\title{
Simulation of the Heat Transfer in the Nanocathode
}

\author{
V.G. Daniov \\ Moscowtechnicaluniversity ofcommunicationsand \\ informatics, \\ Moscowinstitute ofelectronics and mathematics National \\ research university Higher school of economics \\ MTUCI, \\ MIEM NRU HSE \\ Moscow, Russia \\ danilov@miem.edu.ru
}

\author{
V.Yu. Rudnev \\ Moscowtechnicaluniversity ofcommunicationsand \\ informatics, \\ Moscowinstitute ofelectronics and mathematics National \\ research university Higher school of economics \\ MTUCI, \\ MIEM NRU HSE \\ Moscow, Russia \\ vrudnev78@mail.ru \\ V.I. Kretov \\ Moscowinstitute ofelectronics and mathematics National \\ research university Higher school of economics \\ MIEM NRU HSE \\ Moscow, Russia \\ ps-vad@yandex.ru
}

\begin{abstract}
The heat transfer processis simulated in a nano-sized cone-shaped cathode. A model of heat transfer is constructed using the phase field system and theNottingham effect. We considerinfluence of the free boundary curvature and the Nottingham effect on the heat balance in the cathode.
\end{abstract}

Keywords - thermo-field emission, cathode, Nottingham effect, free boundary, phase field system, Stefan-Gibbs-Thomson problem

\section{Introduction and Statement of the Problem}

Our main goal is to simulate the heat transfer ina doped silicon nanocathode. Thecathode has the shape of a blunted cone and the following linear dimensions:

\begin{tabular}{|c|c|}
\hline height of the cathode & $10-15 \mu$ \\
\hline diameter of the cathode base & $\sim 6 \mu$ \\
\hline radius of the cathode vertex rounding & $\sim 15 \mathrm{~nm}$ \\
\hline cathode vertex angle & $\sim 20^{\circ}$ \\
\hline
\end{tabular}

Such a shape of the cathode is specified by the engineering process, see Fig.1.Such cathodes are used in the electron microscope and in other electron devices.

An obstacle for a wide use of this cathode is the instability of electron emission. This instability is in fact caused by the small size of the cathode. The cathode is heated due tothe Joule effect. The current in the cathode is very large and the Joule

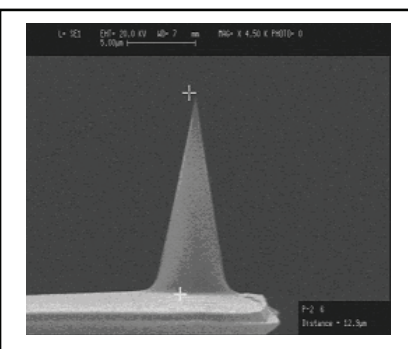
heat can melt the cathode.

Figure 1. REM image of the silicon nanocathode.

The effect of the cathode melting is confirmed experimentally. Namely, the produced molten (liquid) layer does not contain a small region near the vertex of the cathode cone. At the same time, the cathode material remains solid near the base. So we can observe the following sequence of layers: solid, liquid, solid. It is experimentally known that the liquid layer becomes solid after some (unknown) time.

We present an explanation of this fact in this paper. The motion of the free boundary (the interface between the phases) depends on the curvature of the free boundary and the Nottingham effect. Namely, the temperature dependence on the free boundary curvature is determined by the Gibbs-Thomson law $[8,9]$. The Nottingham effect determines the temperature of the cathode vertex under the thermoemission of electrons [1]. More precisely, the Nottingham effect consists in the following. If the temperature of the cathode vertex is higher than the socalled inverse temperature, then the vertex is cooled; if the temperature of the cathode vertex is lower than this inverse temperature, then the vertex is heated.

The mathematical model of the heat transferin the case of fieldemission is known (see [6]),

$$
\rho c(T) \frac{\partial T}{\partial t}=\nabla(\lambda(T) \nabla T)+F, \operatorname{div} j=0 .
$$

Here $T$ isthetemperature, $\rho$ isthedensity, $c$ isthespecificheat, $\lambda$ isthespecific heat capacity, $F$ is the power density of the heat emission under the Joule and Thomson effects, and $j$ is the 
current density. The function $F$ and the current density are determined by formulas

$$
F=\frac{1}{\sigma(T)} j^{2}(r, t)+g(t)\langle j, \nabla T\rangle, \quad j=-\sigma(T)[\nabla u+\alpha(T) \nabla T],
$$

where $\sigma(T)$ isthe specific conductivity, $g(t)$ is the Thomson coefficient, $u$ is the potential of the electric field inside of the cathode, and $\alpha(T)$ is the thermoelectric coefficient.

In our model we use the physical parameters:

\begin{tabular}{|c|c|l|}
\hline$t_{0}$ & $100 \mathrm{c}$ & time scale (time of the experiment) \\
\hline$r_{0}$ & $10^{-5} \mathrm{~m}$ & space scale (size of the cathode) \\
\hline$l$ & $1.64 \cdot 10^{5} \mathrm{~J} / \mathrm{kg}$ & latent heat of melting \\
\hline$c$ & $678 \mathrm{~J} /(\mathrm{kg} \cdot \mathrm{K})$ & Specific heat \\
\hline$\sigma$ & $0.725 \mathrm{~N} / \mathrm{m}$ & surface tension \\
\hline$\rho$ & $2330 \mathrm{~kg} / \mathrm{m}^{3}$ & Density \\
\hline$\mu$ & $0.5 \mathrm{~m} /(\mathrm{c} \cdot \mathrm{K})$ & kinetic coefficient of growth \\
\hline$T_{0}$ & $1700 \mathrm{~K}$ & Melting temperature \\
\hline$k$ & $9.43 \cdot 10^{-5} \mathrm{~m}^{2} / \mathrm{c}$ & Thermal conductivity \\
\hline$\lambda$ & $149 \mathrm{~W} /(\mathrm{m} \cdot \mathrm{K})$ & Specific heat capacity \\
\hline$e$ & $1.602 \times 10^{-19} \mathrm{C}$ & absolute charge of electron \\
\hline$\psi$ & 0.7 & emittance \\
\hline$\sigma_{S B}$ & $5.6704 \cdot 10^{-8} \mathrm{~J} /\left(\mathrm{c} \cdot \mathrm{m}^{2} \cdot \mathrm{K}^{4}\right)$ & Stefan-Boltzmann constant \\
\hline
\end{tabular}

Weconsidera simplifying modificationofthismodelwhich is adapted to the research of silicon small-size cathodes.Namely, the Thomson effect can be neglected because of thep-n conductivity of the silicon emitter. In this case, the contributions of the $\mathrm{p}$ - and n-carriers to the thermoEMF are mutually compensated.We assume that the current densityis constant in the cathode sections thatareorthogonal to the cathode axis. The value of current density was taken approximately from experimental data.

So we reduce system (1), (2) to the one heat equation

$$
\frac{\partial T}{\partial t}-k \frac{t_{0}}{r_{0}^{2}} \Delta T=\frac{t_{0}}{l \rho} F .
$$

Here $r$ is the dimensionless coordinate and $t$ is the dimensionless time. But this is not enough. It is necessary to add the condition at the blunted vertex of the cathode, which corresponds to the Nottingham effect. We also need to include the Gibbs-Thomson and Stefan conditionson the free boundary (the interface between the phases).

We assume that the Gibbs-Thomson condition is satisfied on the free boundary $\Gamma(t)$ (if this free boundary is already generated)

$$
\left.\left(T-T_{0}\right)\right|_{\Gamma(t)}=-\frac{c}{\mu l} \mathbf{v}-\frac{\sigma c T_{0}}{l^{2} \rho} K,
$$

where $\mathbf{v}$ is the normal velocity of the free boundary and $K$ is the principle curvature of the free boundary. The normal $\mathbf{n}$ is the outward normal to the interface between the phases (from liquid to solid). Equation (3) determinesthe linear dependence of the temperature on the free boundary curvature and the velocity. If we assume that the free boundary $\Gamma(t)$ is determines by the function $r=r(t)$, then we have $\mathbf{v}=\left(r_{0} / t_{0}\right) r^{\prime}(t)$ and $K=1 /\left(r_{0} r(t)\right)$.

Besides it is necessary to assume that the Stefan condition is satisfied on the free boundary

$$
\left.k\left[\frac{\partial T}{\partial \mathbf{n}}\right]\right|_{\Gamma(t)}=-\mathbf{v} .
$$

If we get $\frac{c}{\mu l} \frac{r_{0}}{t_{0}} \ll 1$ and $\frac{\sigma c T_{0}}{l^{2} \rho} \frac{1}{r_{0}} \ll 1$ in (3), then condition (3) becomes the usual widely known condition

$$
\left.T\right|_{\Gamma(t)}=T_{0}
$$

In our case, $\frac{c}{\mu l} \frac{r_{0}}{t_{0}} \rightarrow 0$ and $\frac{\sigma c T_{0}}{l^{2} \rho} \frac{1}{r_{0}} \gg 1$. So we obtain from (2)

$$
\left.\left(T-T_{0}\right)\right|_{\Gamma(t)}=-\frac{\sigma c T_{0}}{l^{2} \rho} K .
$$

At the blunted vertex of the cathode $\left(r=R_{0}\right)$ we use the equation(see [7]

$$
\left.\frac{\lambda}{r_{0}} \frac{l}{c} \frac{\partial T}{\partial r}\right|_{r=R_{0}}=\left.\frac{j}{e} E\right|_{r=R_{0}}-\left.\psi \sigma_{S B}\left(\frac{l}{c}\right)^{4} T^{4}\right|_{r=R_{0}}
$$

Here $E$ is the energy of the emission electrons. In the righthand side of equation (6), the first term determines theNottingham effect and the second term determines the additional radiation condition. To derive the function $E$ we use the approximating from [1].

On theother outer boundaries of theblunted-cone cathode, we use Neumann-type boundary conditions.

Finally, we obtain problem (2), (4), (5) with condition (6), which models the thermo-field emission under our assumptions. As was mentioned above, the liquid layer can be produced. This fact means that the domain of our problem can change in time. This leads to serious obstacles for the numerical simulation. To avoid these obstacles, we use a regularization of problem (1), (4), (5). This regularization is the phase field model (see [2-4])

$$
\frac{\partial \theta}{\partial t}-k \frac{t_{0}}{r_{0}^{2}} \Delta \theta=-\frac{1}{2} \frac{\partial \varphi}{\partial t}+\frac{t_{0}}{l \rho} F,
$$




$$
\varepsilon \frac{c}{\mu l} \frac{r_{0}}{t_{0}} \frac{\partial \varphi}{\partial t}-\varepsilon \frac{\sigma c \theta_{0}}{l^{2} \rho} \frac{1}{r_{0}} \Delta \varphi=\frac{g(\varphi)}{\varepsilon}+\chi\left(1-\varphi^{2}\right)\left(\theta-\frac{c}{l} T_{0}\right) .
$$

$$
\text { Here } \quad \chi=\sqrt{2} / 5 . \quad \operatorname{Inmodel}(7)
$$

thestateofthesystemisdeterminedbythe dimensionlessparameter $\varphi=\varphi(r, \phi, \vartheta, t)$ (the so-called order function) in addition to the usualphysicalparameters (temperature, density, etc.). The function $g(\varphi)$ isthederivativeofthepotential $W(\varphi)$. This potentialissymmetricwith respect tozeroandhastwominima at the points $\varphi= \pm 1$. In the simple case, we have $g(\varphi)=\varphi-\varphi^{3}$. The function $\theta=(r, \phi, \vartheta, t, \varepsilon)$ is a regularization of the temperature $T$ in (2), and $\varepsilon$ is the regularization parameter.

Namely, if we formally let $\varepsilon \rightarrow 0+0$, thenequation (7) becomesthe heat equation (2) and equation (8) gives condition (3) (or condition (5) as a particular case of (3)) and condition (4).In general, the limit transition as $\varepsilon \rightarrow 0$ from the phase field system (7), (8) to problem (2), (4), (5) is nontrivial. This question is discussed in $[5,7]$.

System (7), (8) is supplemented with the boundary (Neumann-type) conditions. At the blunted vertex of the cathode ( $r=R_{0}$ ) we use equation(6) for the function $\theta$.

In our model,we take into account the fact that the coefficients $k, \rho, \lambda, c$ depend on the temperature.To obtain the effects of melting and solidification,we also introduce the condition of generation of aseed of the liquid phase in the solid phase and vice versa.

\section{Numerical solution and programming}

Thespecialfeaturesofsystem (7), (8) are the following. First: thecoefficient at the time derivative in (8) is $\frac{c}{\mu l} \frac{r_{0}}{t_{0}} \approx 10^{-11}$. Thisfactmeansthatthe motion of the free boundary $\Gamma(t)$ depends on the freeboundaryvelocity much lesser than on the free boundary curvature, see (5).Second: thecoefficient (thermalconductivity) $k t_{0} / r_{0}^{2}$ isverylarge $\left(\approx 10^{7}\right)$ in (7).This fact means that the temperature rapidly stabilizes in a small volume.

Theaforesaidmeansthatit is necessary to solve system (5), (6) toconstruct the solution on a long time interval.Thisisa veryserious problem. Thefactis thatequation (8) is nonlinear and its "innerinstability" generates nonlinear waves.Thisfactleadsto

thegenerationoftheinterfacebetweenthephases

boundary).However, the final form(5) of the parameter (3) shows that equation (8) has a stationary solution at the given temperature.

Thisfactallowsone to solve system (7), (8) byusing an iterationalgorithm. We use the standard implicit difference scheme.For every time step $k$, we first solve the linearized equation (8). The sweepingisexecutedforgiven ( $\left.n_{1}\right)$ times.Sowefindthestationarysolution $\varphi^{k+1}$ ofequation (8) at the given temperature $\theta^{k}$. Next we derive the heat equation (7) with the function $\varphi^{k+1}$. The sweepingisalso executedforgiven $\left(n_{2}\right)$ times. Sowefindthestationarytemperature $\theta^{k+1}$.

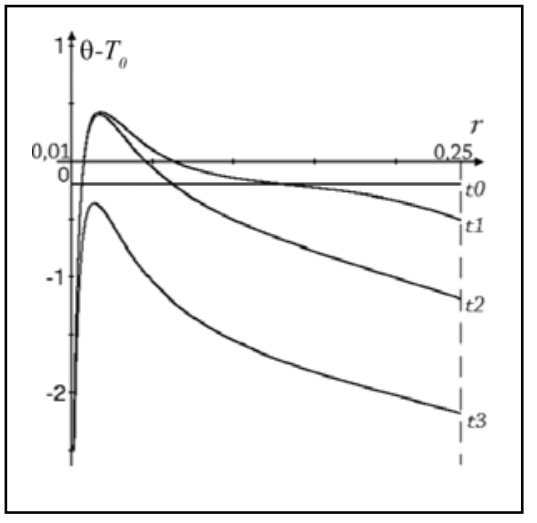

Thecomputerprogramwasproducedtoderivesystem (7), (8)

Figure 2. Dynamycs of the deviation of the dimensionless temperature $\theta-T_{0}: t=t_{0}=0, t=t_{1}=0,2 \cdot 10^{-2}$ (At this instant of time liquid phase is generated), $t=t_{2}=1,5 \cdot 10^{-2}$. (At this instant of time the left free boundary $r=r_{1}(t)$ changes moving direction), $t=t_{3}=4,5 \cdot 10^{-2}$. (At this instant of time the free boundaries merge), $\varepsilon=0.03$.

numericallybythe abovealgorithm. Thisprogramallows one to varythevalues of the system parameters and the computational algorithm. Forexample, iftheStefancondition (4) and theGibbsThomsoncondition

(3)donotcontainlargeorsmallparameters, thenwecanassume $n_{1}=n_{2}=1$.

\section{Simulation Results}

In Figs. 2, 3, 4, we present the results of numerical simulation of the liquid layer generation and the motion of the free boundaries.

In Fig. 2,the deviation of the dimensionless temperature $\theta-T_{0}$ is shown. At the initial time moment, the dimensionless temperature is equal to a negative constant,see Fig. 2, $t_{0}=0$. We also assume that the cathode is solid at the initialinstant of time $t=0$. This means $\left.u\right|_{t=0}=-1$, see Fig. 3 . The boundary conditions used here mean that the cathode vertex is cooled because of the Nottingham effect, while the lower base is cooled due to the Neumann-type conditions $\left.\frac{\partial T}{\partial r}\right|_{r=R}=-\alpha\left(T_{R}-T\right)$, where $T_{R}$ is a room temperature.

Figure 3. Dynamics of the order function $u$. $t=t_{0}=0$, $t=t_{1}=0,2 \cdot 10^{-2} \quad$ (At this instant of time liquid phase is generated), $t=t_{2}=1,5 \cdot 10^{-2}$, $t=t_{3}=4,5 \cdot 10^{-2}$. (At this

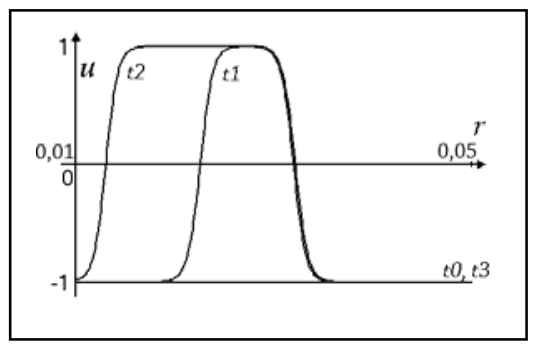


instant of time the free boundaries merge), $\varepsilon=0.03$

Because of the Joule heat, the temperature increases in the middle of the cathode, while the temperature decreases near the boundary points $r=R_{0}$ and $r=R$. because of the Nottingham effect and the cooling of the cathode base.

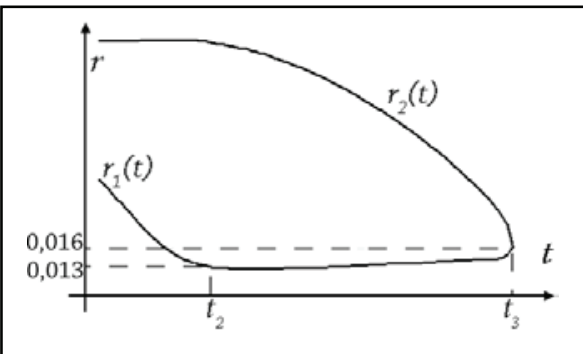

Figure 4. Trajectories of the free boundaries $r=r_{1}(t)$ and $r=r_{2}(t)$. $t=t_{2}=1,5 \cdot 10^{-2}$. (The free boundary $r=r_{1}(t)$ changes moving direction), $t=t_{3}=4,5 \cdot 10^{-2}$. (The free boundaries $r=r_{1}(t)$ and $r=r_{2}(t)$ merge).

So the temperature profile has maximum inside of the domain,where the temperature is higherthan the melting temperature $T_{0}$, see Fig. $2, t=t_{1}$. In the heated domain of the cathode, the liquid layer is generated, see the profile of the order function in Fig. 3, $t=t_{1}$. Because the heat outflow due to the Nottingham effect increases with increasing temperature, the heating is changed by the cooling as the temperature attains some maximum value. In Fig. 4 the trajectories of the free boundaries are plotted, the lower curve corresponds to the left free boundary $r=r_{1}(t)$ and the upper curve corresponds to the right free boundary $r=r_{2}(t)$. One can see that the melting region (distance between curves, see Fig.4) the melting region first begins to expand (the distance between the curves along the vertical increases) and then decreases to zero, $t=t_{3}$.

\section{REFERENCES}

[1] J. Paulini, T. Klein, and G. Simon,"Thermo-field emission and the Nottingham effect," J. Phys. D: Appl. Phys. 26 (1993) Printed In the UK, pp.1310-1315.

[2] G. Caginalp, "An analysis of a phase field model of afree boundary," Arch. Rat. Mech. Anal. 92 (1986), pp.205-245.

[3] G. Gaginalp, Arc. Rational Mech. Anal., 92, 205 (1986).

[4] G. Gaginalp, in Applications of Field Phase Theory to Statistical Mech., v.216 of Lecture Notes in Physics, Springer, Berlin, p. 216.

[5] V.G. Danilov,

G.A. Omel'yanov, E.V. Radkevich,"Hugoniot type conditions and weak solutions to the phase field system," Eur. Journ. Appl. Math. (1999), 10, pp.55-77.

[6] D.V. Glazanov, L.M. Baskin, G.N. Fursey, "Kinetics of pulse heating of sharp-shaped cathode with real geometry by emission current of a high density."Journal of Tech. Phys., v. 59, n.5, (1989) pp. 60-68. English translation in Journal of Tech. Phys.

[7] P.I. Plotnikov and V.N.Starovoitov, "Stefan problem as the limit of the phase field system." Differential Equations 29 (1993), 461-471.

[8] J.W. GribbsCollectedWorks, YaleUniversityPress, NewHaven, 1948.

[9] C.M. Elliot, J.R. Ockendon, "Weak and Variational Methods for Moving Boundary Problems," Pitman, Boston, 1982. 